\title{
An association between chromosomal abnormalities in rapidly frozen 2 -cell mouse embryos and the ice-forming properties of the cryoprotective solution
}

\author{
J. M. Shaw, I. Kola, D. R. MacFarlane* and A. O. Trounson \\ Centre for Early Human Development, Monash University, Monash Medical Centre, Clayton, \\ Victoria 3168, Australia; and ${ }^{*}$ Chemistry Department, Monash University, Clayton, Victoria 3168 , \\ Australia
}

\begin{abstract}
Summary. This paper investigates the effect of straw handling on the viability of 2-cell mouse embryos rapidly frozen in dimethyl sulphoxide (DMSO) solutions. During the brief ( $3 \mathrm{~min}$ ) equilibration step, straws were either rotated periodically to keep the embryos in suspension, or kept still to allow the embryos to settle onto the inner surface of the straw. The effects of these straw movements were tested with cryoprotectant solutions containing $1 \cdot 5,3 \cdot 0$ or $4 \cdot 5 \mathrm{M}$-DMSO. Rapidly cooled straws containing $4.5 \mathrm{M}$ DMSO vitrify throughout on cooling, but ice forms on warming. The survival and normality of embryos frozen in 4.5 M-DMSO was not influenced by straw handling as $91-92 \%$ formed blastocysts in vitro, $77-78 \%$ formed normal fetuses, and no chromosomal rearrangements were observed. In solutions containing $<4.5 \mathrm{M}-\mathrm{DMSO}$ ice formation occurred throughout (1.5 M-DMSO), or in parts (3.0 M-DMSO) of the cryoprotectant during cooling. The viability of embryos frozen in 3.0 or $1.5 \mathrm{M}$-DMSO solutions was reduced both in vitro and in vivo and structural chromosome aberrations, predominantly tri- and quadri-radial rearrangements, were observed. The reduction in embryo viability, and the chromosomal damage was particularly pronounced in embryos frozen in $3.0 \mathrm{M}$-DMSO in straws which were rotated during the equilibration step ( $47 \%$ blastocysts, $15 \%$ fetuses, $77 \%$ chromosome rearrangements). The results indicate that rapid freezing of 2-cell mouse embryos in $4.5 \mathrm{M}$-DMSO is safe and efficient, whereas freezing at lower DMSO concentrations is associated with severe chromosome damage, and reduced viability in vitro and in vivo.
\end{abstract}

Keywords: dimethyl sulphoxide; rapid freezing; ice formation; handling; chromosome rearrangements; mouse; embryos

\section{Introduction}

Cryopreservation is used in animal husbandry and human in-vitro fertilization programmes to allow the storage of surplus embryos until they are needed. Ideally the freeze-thaw procedure should not cause any loss of viability, or lead to an increased incidence of genetic aberrations, fetal malformations or losses. Methods which use cryoprotectant agents such as dimethyl sulphoxide (DMSO) and slow cooling to low subzero temperatures (Whittingham et al., 1972; Wilmut, 1972), have been used with success for the cryopreservation of embryos from a wide range of species. Slow cooling methods are still in use and provide a reference against which other freezing methods can be compared.

The major disadvantage of the slow cooling method for routine application to embryo freezing is its time-consuming nature. As a result research has been directed towards the development of faster and cheaper freezing methods. There are now a number of 'rapid' freezing protocols, which 
are achieving survival rates approaching those for embryos frozen by slow cooling. However, the safety of most of these rapid freezing methods has not been thoroughly investigated, even though it is important that any deleterious effects on embryonic normality and viability be established before they are more widely adopted. In one study, Bongso et al. (1988) found that up to $3.5 \%$ of rapidly frozen mouse embryos had chromosomes with structural rearrangements. These chromosomal rearrangements were observed in embryos frozen in $3.0 \mathrm{M}$-DMSO but not in embryos frozen in $3.0 \mathrm{M}$-propanediol. We have frozen mouse embryos by rapid freezing in solutions containing up to 4.5 m-DMSO (Shaw et al., 1988; Shaw \& Trounson, 1989) and were therefore keen to ascertain whether the damage observed by Bongso et al. (1988) was linked with the use of high concentrations of DMSO. This paper investigates the effects of the DMSO concentration on the chromosomal integrity and in-vitro and in-vivo survival rates of rapidly frozen 2-cell mouse embryos. The effect of the position of the embryo in the cryoprotectant at the time of freezing was also assessed since we had observed that, after rapid cooling, the 3.0 M-DMSO solution appeared to vitrify at the edge (close to the straw wall), while having a central core of ice.

\section{Materials and Methods}

\section{Source and in-vitro culture of embryos}

Two-cell embryos were collected from the oviducts of 5-6-week-old superovulated F1 (C57BL/6J $\times \mathrm{CBA}$ / CaHWehi) hybrid mice, mated to males of the same strain. The mice were superovulated with 5 i.u. pregnant mares' serum gonadotrophin (PMSG) (Folligon: Intervet, Lane Cove, NSW, Australia) followed $48 \mathrm{~h}$ later with 5 i.u. human chorionic gonadotrophin (hCG) (A.P.L. Ayerst Labs, Parramatta, NSW, Australia). The embryos were flushed $44 \pm 1 \mathrm{~h}$ after hCG using Medium M2 (Quinn et al., 1982), $\mathrm{pH} 7 \cdot 2-7 \cdot 4$, at room temperature. All cryoprotectant and wash solutions for embryos were made up in Medium M2.

\section{Freezing and thawing procedures}

Rapid cooling. The cryoprotective solutions contained $0 \cdot 25$ M-sucrose (Analytical Grade, BDH, Kilsyth, Victoria, Australia), $8 \mathrm{mg}$ crystalline bovine serum albumin/ml (BSA) (Bovine Albumin, Commonwealth Serum Laboratories, Melbourne, Victoria, Australia), and 1.5, 3.0 or 4.5 M-DMSO (BDH), in Medium M2. The cryoprotectant solutions were adjusted to $\mathrm{pH} 7 \cdot 2-7.6$ with $\mathrm{NaOH}$. Clear plastic 0.25 -ml bovine insemination straws (IMV AA 20l, Victorian Artificial Breeders, Bacchus Marsh, Victoria, Australia) were used as supplied by the manufacturer. They were not sterilized or washed before use. Embryos were transferred with a minimal amount of Medium M2 into 30-40 $\mu$ l cryoprotectant solution in the insemination straw, using a finely drawn glass pipette (usually 20 embryos in each straw). The straws were heat-sealed at one end, laid horizontally on the bench, and after 3 min gently but rapidly lowered into liquid nitrogen. Inserting the embryos and sealing the straws takes only $15-30 \mathrm{sec}$ and if the straws are not moved again during the equilibration period, embryos will settle against the wall of the straw. If the straws are moved (rotated) during the equilibration period the embryos may become suspended in the cryoprotectant solution and not be in contact with the wall of the straw at the time of freezing. The normality and viability of embryos frozen in straws which had not been moved during the equilibration period were compared with those of embryos from straws that had been periodically rotated to keep the majority of the embryos in suspension at the time of freezing. The position of the embryos, resting on the wall of the straw or suspended in solution away from the straw wall, was confirmed by examination under a dissecting microscope before plunging the straw into liquid nitrogen. In addition to the usual $3 \mathrm{~min}$ equilibration, some embryos exposed to $4.5 \mathrm{M}$-DMSO were equilibrated for only $2 \mathrm{~min}$ before being plunged into liquid nitrogen; this aimed to establish whether any damage caused by the use of $4.5 \mathrm{M}$-DMSO would be modified by using a shorter equilibration time. All work was carried out at room temperature $\left(20-22^{\circ} \mathrm{C}\right)$. The embryos were stored in liquid nitrogen for a minimum of $24 \mathrm{~h}$ before thawing. Straws were thawed by immersion in a $35-37^{\circ} \mathrm{C}$ waterbath. As soon as the cryoprotectant solution had melted $(3-6 \mathrm{sec})$ the straw was removed from the waterbath and the thawed solution expelled into $2 \mathrm{ml}$ Medium M2 containing $0.25 \mathrm{M}$-sucrose at room temperature. After $10 \mathrm{~min}$ the embryos were collected in a minimal amount of solution and transferred to $2 \mathrm{ml}$ Medium $\mathrm{M} 2$ to remove the sucrose. After $10 \mathrm{~min}$ in Medium M2, the embryos were transferred to droplets of culture medium. Embryos were considered to have survived the freezing procedure if one or both blastomeres were intact when placed in culture.

Slow cooling. To provide a comparison with rapid freezing, 2-cell embryos were frozen and thawed by a conventional slow cooling method. Embryos were exposed stepwise for $10 \mathrm{~min}$ to $0.5,1.0$ and $\mathrm{I} \cdot 5 \mathrm{M}$-DMSO in Medium M2 (with $4 \mathrm{mg} \mathrm{BSA} / \mathrm{ml}$ ), inserted into insemination straws, and cooled horizontally in the liquid nitrogen vapour of a Cryogenics freezer (Cryogenics, Soquel, CA, USA). Straws were cooled at $1-2^{\circ} \mathrm{C} / \mathrm{min}$ from ambient temperature to $-7^{\circ} \mathrm{C}$. The straws were seeded at $-7^{\circ} \mathrm{C}$, held for $10 \mathrm{~min}$ and then cooled to $-80^{\circ} \mathrm{C}$ at $0.3^{\circ} \mathrm{C} / \mathrm{min}$, before plunging 
into liquid nitrogen. Embryos were thawed from $-80^{\circ} \mathrm{C}$ to $+4^{\circ} \mathrm{C}$ at $8^{\circ} \mathrm{C} / \mathrm{min}$ in a Planer freezer (Planer, Sunbury on Thames, UK), and DMSO was removed stepwise at 10 -min intervals by transferring embryos to $1.5,1.0,0.75,0.5$, 0.25 and $0 \mathrm{M}$-DMSO in Medium M2. Embryos were washed in Medium M2 and then cultured in vitro, processed for chromosome analysis or transferred to the oviducts of recipients.

\section{Development in vitro}

The in-vitro development of embryos rapidly cooled after equilibration in 1.5, 3.0 and 4.5 M-DMSO with or without straw handling, was compared with that of slowly cooled embryos, embryos placed in culture immediately after collection (controls), and embryos exposed to the same cryoprotectant solutions, and handling procedures but not frozen (solution controls).

Embryos were cultured at $37^{\circ} \mathrm{C}$ in $30-\mathrm{mm}$ plastic Petri dishes (K-Line, Labco, Cheltenham, SA, Australia) in $100 \mu \mathrm{l}$ droplets of Whittingham's T6 medium (Quinn et al., 1982), supplemented with $10 \%$ fetal calf serum (Commonwealth Serum Laboratories), under light-weight paraffin oil (BDH) in an humidified atmosphere of $5 \%$ $\mathrm{CO}_{2}$ in air. Development in culture was assessed by daily microscopic examination over a 6-day period. Two end points were used, blastocyst formation, defined as the presence of a blastocoele by the 4th day in culture, and hatching, defined as the partial or complete escape of the embryo from the zona pellucida by the 6th day in culture. Intact and damaged embryos were cultured together.

\section{Development in vivo}

The development in vivo of embryos rapidly cooled in 1.5, 3.0 and 4.5 M-DMSO, with or without straw rotation, was compared with that of embryos frozen by slow cooling and freshly collected untreated control embryos.

Thawed 2-cell embryos were transferred to the oviducts of pseudopregnant recipients after 2-5 h incubation in vitro. The recipients were 3-4-month-old FI (C57BL/6J $\times$ CBA/CaHWehi) hybrid females mated to vasectomized male mice. Recipient females were examined in the morning for the presence of a copulation plug, and transfers were carried out on the same day (Day l) under tribromoethanol anaesthesia. Each pseudopregnant recipient received frozen-thawed embryos on one side and either frozen-thawed or control embryos on the contralateral side. A maximum of 6 embryos was transferred per side. Individual mice were identified by ear clipping. The recipients were autopsied on Day 15 of gestation, and the total number of implantation sites and normal fetuses in each horn was recorded.

\section{Chromosome analysis}

The chromosomal integrity of embryos rapidly frozen following a 3-min equilibration period in $1.5,3.0$ and $4.5 \mathrm{M}$ DMSO, with or without straw rotation, was compared with that of embryos frozen by slow cooling $(\mathrm{N}=95)$, untreated (control) embryos $(\mathrm{N}=75)$, and embryos placed in $3.0 \mathrm{M}$-DMSO for $3 \mathrm{~min}$ but not frozen-thawed (solution control) $(\mathrm{N}=73)$. Embryo collection, and the addition of colcemid, was timed relative to the hCG injection, excluding the time of frozen storage. In these experiments embryos were collected and frozen at $44 \pm 1 \mathrm{~h}$ after hCG and colcemid $(0 \cdot 1 \mu \mathrm{g} / \mathrm{ml})$ was added to all cultures at $49 \mathrm{~h}$ after hCG. Embryos start cleaving from $2-4$ cells at around $50 \mathrm{~h}$ after hCG in our laboratory. After $12 \mathrm{~h}$ exposure to the colcemid, embryos were placed in hypotonic solution ( $1 \%$ trisodium citrate in water) until swollen $(3-15 \mathrm{~min})$. The swollen embryos were individually placed on a clean glass microscope slide in a minimal amount of fluid and then spread using drops of acetic acid and methanol $(1: 3, v / v)$ (Kola et al., 1986). The slides were stained in Giemsa (Gurr), and examined under oil immersion $(\simeq \times 1250$ magnification) for structural chromosome aberrations. Slides were coded, and scored independently by two of us (J.M.S. and I.K.). This study concentrated on structural rather than numerical chromosomal anomalies. A preparation was considered to have errors if breaks, gaps or rearrangements were seen, even when the full complement of chromosomes could not be found. A chromosome preparation was considered to be normal only if the full complement of intact chromosomes was present; if any chromosomes were missing from an otherwise 'normal' spread the preparation was classified as non-scorable. Only chromosome spreads which both independent workers agreed were chromosomally aberrant were classified as aberrant; all other spreads were considered to be non-scorable.

\section{Scanning electron microscopy of the ice structure in frozen straws}

To establish whether ice crystal sizes were uniform within a straw, we filled straws with $40 \mu 1$ cryoprotectant solution $(1.5,3.0$ or $4.5 \mathrm{M}$-DMSO) and plunged them in the usual way into liquid nitrogen. The frozen straws were inserted into a copper block kept at $-196^{\circ} \mathrm{C}$, and very rapidly transferred to a liquid nitrogen vapour cooled $\left(-150^{\circ} \mathrm{C}\right)$ sputter coater (Emscope SP 2000 Cryo-preparation unit). The straws were fractured after evacuating the chamber to 0.01 Torr, and then gold coated under an argon atmosphere at 0.05 Torr. The coated straws were transferred, under vacuum, to a liquid nitrogen cooled Emscope Cryostage fitted to an Hitachi S570 scanning electron microscope. The freeze fracture patterns of all three DMSO concentrations were examined, at up to $\times 20000$ magnification, with particular attention to any differences between the periphery and the centre of the straw. 


\section{Statistical analysis}

The overall significance of treatments in these experiments was determined using linear-logistic models (equivalent to analysis of variance) using the GLIM statistical package (version 3.77) (Baker \& Nelder, 1978), assuming a binomial error distribution. These analyses used a survival analysis approach (Cox \& Oakes, 1984), comparing the agespecific survival rates (i.e. probability that an embryo will survive to the next stage, given it survived to the beginning of the current stage). Further comparisons between individual treatments were completed by $\chi^{2}$ analysis of $2 \times 2$ tables.

\section{Results}

\section{Embryo survival and development}

Embryos recovered from the freezing straws of all experiments were classified as intact, semiintact or dead. The proportions of embryos in each category were, in the slow cooling group $67 \%$, $14 \%$ and $19 \%$ respectively, and in the rapidly frozen groups $98,1 \cdot 7,0.4 \%(4.5 \mathrm{M}$, rotated, $3 \mathrm{~min})$, $99,0.5,0.5 \%(4.5 \mathrm{M}$, non-rotated, $3 \mathrm{~min}), 99,0 \cdot 6,0.3 \%(3.0 \mathrm{M}$, rotated $), 97,2,1 \%(3.0 \mathrm{M}$, nonrotated), $92,4,4 \%(1.5 \mathrm{M}$, rotated), and $88,8,4 \%(1.5 \mathrm{M}$, non-rotated). The results below are presented per embryo frozen rather than per embryo recovered because some embryo losses could be due to the embryo and zona being damaged beyond recognition by the time the straw contents were examined (Shaw et al., 1988).

\section{Development in vitro}

Similar results were obtained in all the control groups (Table 1). Non-frozen 2-cell embryos developed at high rates $(90-97 \%)$ to the blastocyst stage in culture and there was no significant difference between those exposed to the cryoprotective solutions and those which were not (G2(1) $=0.12 ; P>0.2)$ and among the solution controls developmental rates were not affected by DMSO concentration $(\mathrm{G} 2(2)=4.86 ; P>0.05)$ or rotation $(\mathrm{G} 2(1)=3.47 ; P>0.05)$.

Table 1. In-vitro development of frozen-thawed and solution control 2-cell mouse embryos

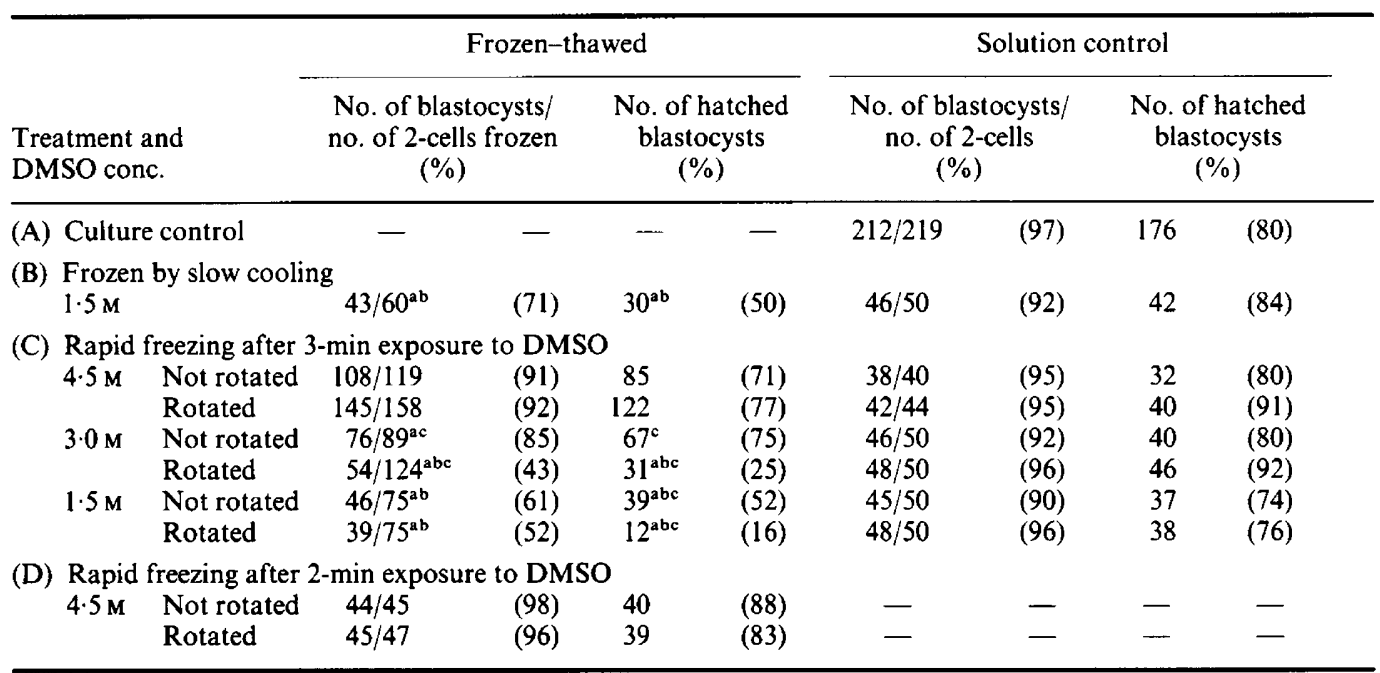

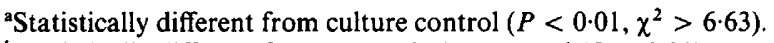

'Statistically different from own solution control $(P<0.01)$.

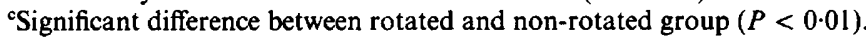


Among the frozen-thawed embryos, development to the blastocyst or hatched blastocyst stage varied depending on the DMSO concentration in the cryoprotectant solution and whether the straw was rotated or not during the equilibration step before plunging into liquid nitrogen (overall G2(2) $=12.79, P<0.01$; effect of rotation G2(1) $=25.23, P<0.001$; effect of DMSO concentration G2(2) $=7.74, P<0.01$ ) (Table 1 ).

Freezing in $4.5 \mathrm{M}$-DMSO did not significantly reduce the developmental ability of 2-cell embryos to form blastocysts or hatch, as compared with their non-frozen solution control, regardless of straw movements or the time of exposure ( 2 or $3 \mathrm{~min}$ ). Blastocyst formation was significantly reduced in most groups in which embryos were frozen and thawed in 3.0 or 1.5 M-DMSO (Table 1). The reduction was greater in straws which had been rotated during the equilibration period, keeping the embryos suspended in the cryoprotectant at the time of freezing. The detrimental effects of straw rotation in $3.0 \mathrm{M}-\mathrm{DMSO}$ was only evident after the first 2 days in culture. The initial cleavage and compaction of embryos from rotated straws was identical to that of non-frozen culture controls, but only $43 \%$ of these embryos developed to the blastocyst stage in vitro (Table 1 ).

The proportion of embryos frozen by slow cooling in $1.5 \mathrm{M}$-DMSO which developed to the blastocyst or hatched blastocyst stage in vitro was significantly lower than for embryos frozen in 4.5 M-DMSO, their own solution control group, and the culture control embryos $(P \leqslant 0.01$ in all cases, by $\chi^{2}$ tests).

\section{Development in vivo}

Only intact 2-cell embryos were selected for transfer, as we have found that damaged embryos implant but rarely form fetuses in vivo. As a result, the proportion of frozen-thawed embryos suitable for transfer varied from $54 \%$ for embryos frozen by slow cooling to $90 \%$ or more for embryos frozen rapidly in 3.0 and $4.5 \mathrm{M}$-DMSO. A total of 38 slow cooled, 248 control, and 445 rapidly frozen embryos were transferred to 67 recipient mice (Table 2). Of these all but one became pregnant. The data from this mouse which received 6 control embryos and 6 frozen in $3 \cdot 0 \mathrm{M}-\mathrm{DMSO}$ (rotated straws) were excluded from further analysis.

Among the 3 control groups, there was no significant difference in the age specific survival rates (implantation sites/embryos transferred and normal fetuses/implantations) $(\mathrm{G} 2(2)=5 \cdot 65$, $P>0.05$ ) (Table 2). However, comparing the results with those for the frozen-thawed embryos, there were significant effects of DMSO $(\mathrm{G} 2(4)=30.96, P<0.001)$ and rotation $(\mathrm{G} 2(2)=9 \cdot 12$, $P<0.01)$, and the effect of rotation differed with different DMSO concentrations $(\mathrm{G} 2(2)=12 \cdot 38$, $P<0.001)$. The sources of these significant interaction effects are discussed individually below.

The implantation rate of embryos transferred to pregnant recipients was $>85 \%$ in all nonfrozen control groups, and varied from 47 to $96 \%$ for frozen-thawed embryos. Only one treatment, freezing and thawing in $3.0 \mathrm{M}$-DMSO rotated straws, significantly reduced the implantation rate below that of the contralateral controls $\left(\chi^{2}=30.9, P<0.001\right)$ (Table 2). The proportion of implantations which failed to produce viable fetuses was $0-16 \%$ in the control groups, $12-53 \%$ in the rapidly frozen groups and $29 \%$ in the group frozen by slow cooling. The most significant increase in resorptions relative to their contralaterally transferred non-frozen controls occurred in the rotated straws with 1.5 or $3.0 \mathrm{M}$-DMSO $\left(\chi^{2}>8.9, P<0.005\right)$. The proportion of non-frozen control embryos which formed normal fetuses was not significantly different from that of embryos frozen in non-rotated straws in $1.5,3.0$ or $4.5 \mathrm{M}$-DMSO, or rotated straws in $4.5 \mathrm{M}$-DMSO. There was, however, a significant decrease in the proportion of fetuses formed by embryos from rotated straws with 1.5 or $3.0 \mathrm{M}$-DMSO, when compared to fresh control embryos and embryos frozen in 1.5 or $3.0 \mathrm{M}$-DMSO non-rotated straws.

Fetuses with major morphological abnormalities were observed in one control and three rapidly frozen groups (Table 2). The abnormalities were in all instances exencephalies or eye defects. The abnormal fetus in the $3.0 \mathrm{M}$-DMSO rotated group had retarded development of all fore and hind 
Table 2. Viability of rapidly frozen and slow cooled embryos in vivo

\begin{tabular}{|c|c|c|c|c|c|c|c|c|}
\hline \multirow{2}{*}{\multicolumn{2}{|c|}{$\begin{array}{l}\text { Treatment and } \\
\text { DMSO conc. }\end{array}$}} & \multirow{2}{*}{$\begin{array}{l}\text { No. of intact } \\
\text { embryos/no. } \\
\text { of frozen } \\
\text { embryos }\end{array}$} & \multirow{2}{*}{\multicolumn{2}{|c|}{$\begin{array}{l}\text { No. of implantation } \\
\text { sites/no. of } \\
\text { embryos } \\
\text { transferred } \dagger(\%)\end{array}$}} & \multicolumn{3}{|c|}{ No. of fetuses $(\%)$} & \multirow{2}{*}{$\begin{array}{c}\text { Estimated } \\
\text { overall } \\
\text { survival } \neq \\
(\%)\end{array}$} \\
\hline & & & & & & rmal & Abnormal & \\
\hline \multicolumn{9}{|c|}{ Frozen by slow cooling } \\
\hline $1.5 \mathrm{M}$ & Not rotated & $43 / 80$ & $31 / 38$ & $(82)$ & 20 & $(53)$ & 0 & 28 \\
\hline \multicolumn{9}{|c|}{ Rapid cooling after 3-min exposure to DMSO } \\
\hline $4.5 \mathrm{M}$ & Not rotated & $48 / 53$ & $34 / 37$ & $(92)$ & 29 & $(78)$ & 0 & 71 \\
\hline $4.5 \mathrm{M}$ & Rotated & $62 / 69$ & $50 / 52$ & (96) & 40 & (77) & $1(2)$ & 71 \\
\hline Control§ & & $-1-$ & $36 / 40$ & (90) & 35 & (87) & $i(2)$ & 87 \\
\hline $3.0 \mathrm{M}$ & Not rotated & $94 / 97$ & $63 / 70^{b}$ & $(90)$ & $46^{b}$ & (66) & 0 & 64 \\
\hline $3.0 \mathrm{M}$ & Rotated & $88 / 91$ & $25 / 53^{a b}$ & (47) & $5^{\mathrm{a}}$ & b (9) & I (2) & 11 \\
\hline Control§ & & $-1-$ & $102 / 117$ & (87) & 84 & (72) & 0 & 72 \\
\hline $1.5 \mathrm{M}$ & Not rotated & $40 / 50$ & $34 / 39$ & (87) & $20^{\mathrm{b}}$ & (51) & 0 & 41 \\
\hline $1.5 \mathrm{M}$ & Rotated & $44 / 48$ & $33 / 43$ & (76) & $10^{\mathrm{a}}$ & ${ }^{\circ}(23)$ & 0 & 21 \\
\hline Control§ & & $-1-$ & $72 / 85$ & $(85)$ & 58 & $(68)$ & 0 & 68 \\
\hline \multicolumn{9}{|c|}{ Rapid cooling after 2-min exposure to DMSO } \\
\hline $4.5 \mathrm{M}$ & Not rotated & $84 / 85$ & $71 / 78$ & (91) & 60 & $(77)$ & 0 & 76 \\
\hline $4.5 \mathrm{M}$ & Rotated & $82 / 84$ & $52 / 67$ & (78) & 42 & (63) & $2(3)$ & 64 \\
\hline
\end{tabular}

aStatistically different from non-frozen control $\left(P<0.01, \chi^{2}>6.63\right)$.

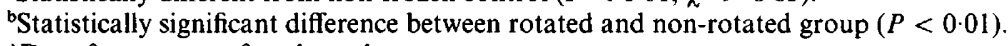

$\uparrow$ Data for pregnant females only.

$\ddagger$ (Intact/frozen embryos) $\times$ (fetuses/no. of embryos transferred to pregnant recipients).

$\S$ Freshly collected 2-cell embryos.

limbs relative to corresponding controls, exencephaly and eye defects. Two intrauterine deaths occurred, and both were in control groups.

\section{Chromosomal analysis}

Chromosomal abnormalities were only found among the frozen-thawed embryos, with the number and severity of the aberrations differing between the groups. There were no structural abnormalities among the scorable chromosome spreads of the cultured control embryos $(\mathrm{N}=54)$, the embryos frozen in $4.5 \mathrm{M}-\mathrm{DMSO}(\mathrm{N}=146$ ), or the $3.0 \mathrm{M}-\mathrm{DMSO}$ solution control group $(\mathrm{N}=67)$. The incidence of abnormalities was significantly increased above the controls only in the groups frozen in $1.5 \mathrm{M}-\mathrm{DMSO}$ (rotated $\chi^{2}=12$, and non-rotated $\chi^{2}=27, P<0.001$ ) and the 3.0 M-DMSO rotated group $\left(\chi^{2}=82, P<0.001\right.$, Table 3 ). The anomalies included tri- and quadriradial rearrangements and gaps. In the most severely damaged embryos up to 6 rearrangements per cell were seen (Fig. 1).

\section{Scanning electron microscopy of the ice structure in frozen straws}

When the freeze-fractured straws were examined under the electron microscope there was no evidence of ice crystal formation in the cryoprotectant solution containing 4.5 M-DMSO. Ice crystals were seen over the whole of the fracture surface of the $1.5 \mathrm{M}$-DMSO solutions, and parts of the fracture surface of the $3.0 \mathrm{M}$-DMSO solutions. Ice formation in 3.0 M-DMSO was not uniform as areas of ice were interspersed with vitrified areas. In some straws the ice crystals were largest in the centre of the straw, and smallest or absent near the edge, while in others there was no difference between the two areas. Straws which had been plunged into liquid nitrogen could be differentiated by their visual appearance. The $4.5 \mathrm{M}$-DMSO solution remained transparent and pink, while the 1.5 M-DMSO solution was opaque and white. The $3.0 \mathrm{M}$-DMSO solution was intermediate between 
Table 3. Chromosomal analyses of 2-cell mouse embryos after overnight culture with colcemid

\begin{tabular}{|c|c|c|c|c|c|c|c|c|}
\hline & \multirow{3}{*}{$\begin{array}{l}\text { Non- } \\
\text { cooled } \\
\text { control }\end{array}$} & \multirow{3}{*}{$\begin{array}{c}\text { Frozen by } \\
\text { slow } \\
\text { cooling }\end{array}$} & \multicolumn{6}{|c|}{ Frozen by rapid cooling } \\
\hline & & & \multicolumn{2}{|c|}{$\begin{array}{c}4 \cdot 5 \mathrm{M} \text {-DMSO } \\
\text { Rotated? }\end{array}$} & \multicolumn{2}{|c|}{$\begin{array}{c}3 \cdot 0 \mathrm{~m} \text {-DMSO } \\
\text { Rotated? }\end{array}$} & \multicolumn{2}{|c|}{$\begin{array}{l}1.5 \mathrm{M} \text {-DMSO } \\
\text { Rotated? }\end{array}$} \\
\hline & & & Not & Yesł & Not & Yesł & No† & Yest \\
\hline No. of 2-cell spreads & 75 & 95 & 60 & 80 & 135 & 185 & 20 & 25 \\
\hline No. of scorable spreads & 54 & 79 & 73 & 73 & 111 & 96 & 13 & 18 \\
\hline No. of normal spreads & 54 & 77 & 73 & 73 & 103 & 22 & 7 & 14 \\
\hline$\%$ Normal & 100 & 97 & 100 & 100 & 93 & $23^{*}$ & $54^{*}$ & $78^{*}$ \\
\hline No. with errors & 0 & 2 & 0 & 0 & 8 & 76 & 6 & 4 \\
\hline Sum quadriradial & 0 & 2 & 0 & 0 & 3 & 79 & 5 & 5 \\
\hline Sum triradial & 0 & 0 & 0 & 0 & 3 & 56 & 2 & 2 \\
\hline Sum breaks or gaps & 0 & 1 & 0 & 0 & 2 & 8 & 2 & 0 \\
\hline
\end{tabular}

†Embryos from straws not moved during the equilibration period.

$\ddagger$ Embryos from straws moved during the equilibration period.

*Statistically different from non-cooled control $(P<0.01)$.

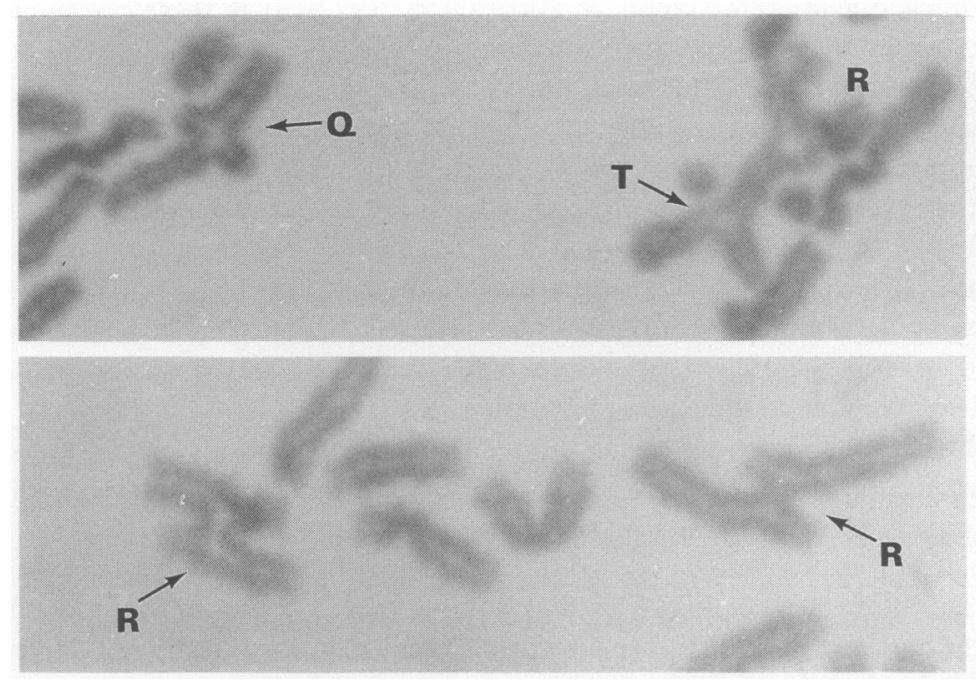

Fig. 1. Examples of chromosomal rearrangements seen in embryos frozen in $3 \cdot 0 \mathrm{M}$-DMSO rotated straws. Quadriradial (Q), triradial (T), other rearrangements (R).

the two and in some straws a distinct transparent pink area could be seen at the edges of the straw. During thawing all straws became opaque and white.

These results show that $4.5 \mathrm{M}$-DMSO solutions vitrify when rapidly cooled and recrystallize when warmed, while 1.5 M-DMSO forms ice when cooled rapidly. The $3.0 \mathrm{M}$-DMSO solution was intermediate between these extremes with some indication, from the electron micrographs, that during rapid cooling the solution close to the plastic straw did vitrify in some straws, even though ice crystals always formed in the centre, further away from the plastic.

\section{Discussion}

We have shown that rapid freezing in solutions containing DMSO can cause severe chromosomal aberrations and reduced embryo viability in vitro and in vivo. The incidence and severity of the 
damage is influenced by both the DMSO concentration and the position of the embryo in the straw at the time of rapid cooling. The highest incidence of chromosomal aberrations occurred among embryos in the $3.0 \mathrm{M}$-DMSO rotated group. This indicates that the chromosomal damage was not caused by the use of high DMSO concentrations, or ice formation during warming. The damage appears more closely linked to factors such as ice crystal formation during rapid cooling.

The finding that the incidence of chromosomal damage manifest as rearrangements was influenced by both the DMSO concentration and straw handling is interesting. For rearrangements to occur there must be chromosome breaks, realignment and fusion of one (tri-radial), two (quadriradial) or more chromosomes. It is possible that DMSO may facilitate these events. High concentrations of DMSO can destabilize DNA (Levine et al., 1963), and RNA (Crawford et al., 1971), and denatured RNA will reaggregate when the DMSO is removed (Birnboim, 1972). DMSO can also transform cell lines (Ashwood-Smith, 1985), and destabilize proteins (Fahy, 1989). As no reduction in viability was observed in any of the solution control groups, and no damage was seen in the group frozen in the highest concentration of DMSO, it seems that the presence of DMSO alone does not determine the extent of the chromosomal damage. Several lines of evidence suggest that the damage may be more closely linked to the freezing properties of the cryoprotectant solution. Bongso et al. (1988) found that rapid freezing in $3.0 \mathrm{M}$-propanediol was not associated with chromosomal damage, even though propanediol, like DMSO and a variety of other cryoprotectants, can denature nucleic acids (Herskovits \& Harrington, 1972). It is therefore interesting that the freezing properties of a $3.0 \mathrm{M}$-propanediol solution (in Medium M2 with $0.25 \mathrm{M}$-sucrose) is more like a $4.5 \mathrm{M}$ - than $3.0 \mathrm{M}$-DMSO solution in that it forms a glass on cooling and freezes on warming (J. M. Shaw, unpublished observations).

Further support for the hypothesis that the chromosomal damage is linked to the freezing properties of the cryoprotectant solution comes from the effects of handling. With 4.5 M-DMSO, ice forms uniformly through the straw upon freezing, and the position of the embryo in the straw does not influence the viability of the embryos after thawing. In 1.5 and 3.0 M-DMSO, for which electron microscopic examination of the straws revealed heterogeneity in the freezing between the peripheral and central regions, the position of the embryos in the straw greatly affected the incidence of chromosomal damage and their viability.

When ice forms the concentration of solutes and cryoprotectants increases as it is predominantly water which is incorporated into the ice crystals. The damage seen in rapidly frozen embryos is, however, unlikely to be explained by such increased solute and cryoprotectant concentrations since slow-cooled embryos are also exposed to increasing solute and cryoprotectant concentrations following ice nucleation. Ice occupies a greater volume than a liquid, or vitrified solution, and it is possible that the pressure exerted by the ice as it expands is greater in the rapidly frozen group. In the straws frozen by slow cooling the ice spreads as a slowly advancing front from the seeded end of the straw. The cryoprotectant bead in rapidly cooled straws first freezes at the ends, then the solution in contact with the plastic straw freezes. The cryoprotectant in the centre of the straw freezes last, and pressure may build up in this region since it is surrounded by an already frozen/ vitrified shell. We cannot explain why the ice which forms in the 4.5 M-DMSO solution on thawing does not appear to be associated with any damage, although the ice would form as a result of homogeneous nucleation of an already solid (vitrified) medium, and may be cubic rather than hexagonal ice (Fahy, 1989). It is also possible that the behaviour of cryoprotectant solutions external to the embryo are not representative of the intracellular environment. We have not, however, attempted to evaluate whether the intracellular components of the embryos vitrify or freeze. Rall (1987) placed embryos in cryoprotectant solutions that vitrified on cooling, and then evaluated the effect of the warming rate. Slow warming rates were associated with ice formation, and ice formation was avoided with the faster warming rates. Embryo survival was uniformly high with the fast warming rates. With the slower warming rates the survival rates ranged from 0 to $90 \%$ depending on the composition of the cryoprotectant solution (VS 1, VS 2 and VS 3) (Rall, 1987). The reasons for these differences were not established. 
The observation that embryo freezing can cause chromosomal damage which does not lead to immediate cell death has serious implications. Exposure of embryos to a variety of teratogens and carcinogens can lead to a similar high incidence of chromosomal rearrangements. For example preimplantation mouse embryos exposed in vivo to cyclophosphamide $(40 \mathrm{mg} / \mathrm{kg})$ had a high incidence of chromosomal aberrations ( $56 \%$ aberrant cells), with an average of 1.7 rearrangements per affected metaphase cell (Kola et al., 1986). This is a magnitude similar to that induced in this study in embryos rapidly cooled in rotated straws with $3.0 \mathrm{M}$-DMSO, i.e. $79 \%$ aberrant cells with an average of 1.8 rearrangements per affected metaphase cell. As the incidence of resorptions was significantly increased above that of the controls, but the incidence of fetal abnormalities, on Day 15 of gestation, among fetuses developing from rapidly frozen embryos, or their offspring, was comparable to that of controls, it seems that the majority of damaged embryos fail to develop to term (this study; Shaw \& Trounson, 1989). Whether damage caused by freezing is always lethal is not clear. The unusual abnormalities seen in one of the fetuses in the 3.0 $\mathrm{M}$-DMSO, handled group may be linked to freezing-induced chromosomal damage. If this is the case then it is clear that all freezing methods need to be rigorously tested before use on embryos, in particular human embryos, and those of defined strains of animals.

The rapid freezing method using 4.5 M-DMSO is more efficient than the conventional DMSO slow cooling method tested, in terms of in-vitro and in-vivo development, and did not, under the conditions tested by us, damage the chromosomes. Whether these conditions will be as effective with embryos at other stages of development, or embryos of other species is not certain. It will be impossible to extrapolate until we know more about the causes of the chromosomal damage in frozen-thawed embryos. Clearly in the future new embryo freezing techniques and novel cryoprotectants will need to be tested not solely for their efficacy in terms of the immediate survival, but also in terms of genetic damage.

This paper shows that rapid freezing of early cleavage stage embryos can result in chromosomal aberrations. The damage may be associated with the freezing properties of the cryoprotectant solution since it was only observed in solutions in which ice formed during rapid cooling ( 1.5 and 3.0 M-DMSO). Chromosome damage was not seen in embryos frozen in $4.5 \mathrm{M}$-DMSO, which vitrifies on cooling, irrespective of the position of the embryo in the cryoprotectant solution at the time of freezing. For rapid freezing of mouse embryos, therefore, we recommend the use of $4.5 \mathrm{M}$-DMSO in preference to lower DMSO concentrations as this avoids chromosome damage and removes any effects caused by straw handling.

We thank Mr John Nailon and Mrs Maria Forsyth for assistance with the scanning electron microscopy; Dr G. Shaw for statistical advice and helpful criticism of the manuscript; and Dr P. McCloud of the Monash University Statistical Consulting Service, Monash University, Clayton, Victoria, Australia, for these analyses. This study was supported by an NH \& MRC grant.

\section{References}

Ashwood-Smith, M.J. (1985) Genetic damage is not produced by normal cryopreservation procedures involving either glycerol or dimethyl sulfoxide: a cautionary note, however, on possible effects of dimethyl sulfoxide. Cryobiology 22, 427-433.

Baker, R.J. \& Nelder, J.A. (1978) The GLIM System, Release 3, Generalized Linear Interactive Modelling. Numerical Algorithms Group, Oxford.

Birnboim, H.C. (1972) Denaturation of rat liver ribonucleic acid with dimethyl sulfoxide. Biochemistry, NY 11, 4588-4591.

Bongso, D.V.M., Mok, H., Ng, S.C., Wong, P.C., Sathananthan, H., Ratnam, S. \& Lee, M-N. (1988) Chromosome analysis of two cell embryos frozen by slow and ultrarapid methods using two different cryoprotectants. Fert. Steril. 49, 908-912.

Cox, D.R. \& Oakes, D. (1984) Analysis of Survival Data. (Monographs on Statistics and Applied Probability). Chapman Hall, New York.

Crawford, J.E., Chan, S.I. \& Schweizer, M.P. (1971) MNR studies of organic solvent denatured yeast phenylalanyl transfer RNA at $220 \mathrm{MHz}$. Biochem. Biophys. Res. Commun. 44, 1-7.

Fahy, G.M. (1989) Vitrification. In Low Temperature Biotechnology: Emerging Applications and Engineering Contributions BED-Vol. 10/HTD Vol. 98. Eds J. J. McGrath \& K. R. Diller.

Herskovits, T.T. \& Harrington, J.P. (1972) Solution 
profiles of the nucelic acid bases and related model compounds. Solubility in aqueous alcohol and glycol solutions. Biochemistry, NY 11, 4800-4810.

Kola, I., Folb, P.I. \& Parker, M.I. (1986) Maternal administration of cyclophosphamide induces chromosomal aberrations and inhibits cell number, histone synthesis, and DNA synthesis in preimplantation mouse embryos. Teratog. Carcinog. Mutag. 6, 115-127.

Levine, L., Gordon, J.A. \& Jencks, W.P. (1963) The relationship of structure to the effectiveness of denaturing agents for deoxyribonucleic acid. Biochemistry, NY 2, 168-175.

Quinn, P., Barros, C. \& Whittingham, D.G. (1982) Preservation of hamster oocytes to assay the fertilizing capacity of human spermatozoa. J. Reprod. Fert. 66, 161-168.

Rall, W.F. (1987) Factors affecting the survival of mouse embryos cryopreserved by vitrification. Cryobiology 24, 387-402.
Shaw, J.M. \& Trounson, A.O. (1989) Effect of dimethyl sulfoxide and protein concentration on the viability of two-cell mouse embryos frozen with a rapid freezing technique. Cryobiology 26, 413-421.

Shaw, J.M., Diotallevi, L. \& Trounson, A.O. (1988) Ultrarapid embryo freezing: effect of dissolved gas and $\mathrm{pH}$ of the freezing solutions and straw irradiation. Human Reprod. 3, 905-909.

Whittingham, D.G., Leibo, S.P. \& Mazur, P. (1972) Survival of mouse embryos frozen to $-196^{\circ} \mathrm{C}$ and $-269^{\circ} \mathrm{C}$. Science, $N Y 178,411-414$.

Wilmut, I. (1972) The effect of cooling rate, warming rate, cryoprotective agent and stage of development on survival of mouse embryos during freezing and thawing. Life Sci. 11, 1071-1079.

Received 16 December 1989 